\title{
On Multi-Consensus and Almost Equitable Graph Partitions *
}

\author{
Salvatore Monaco ${ }^{\text {a }}$, Lorenzo Ricciardi Celsi ${ }^{a}$, \\ ${ }^{a}$ Dipartimento di Ingegneria Informatica, Automatica e Gestionale Antonio Ruberti, \\ Università di Roma La Sapienza, via Ariosto 25, 00185 Roma, Italy
}

\begin{abstract}
This paper deals with multi-consensus in multi-agent systems, focusing on the relationship between multi-consensusability and the underlying digraph topology. In particular, the topological arrangement of the network nodes naturally suggests distinguishing among, on the one hand, separate independent groups of agents agreeing internally and, on the other hand, a dependent common subgraph whose internal consensuses can be computed as a convex combination of the different consensuses achieved by the previously mentioned independent groups. The distinct achieved consensuses are as many as the number of groups of agents defining cells of a suitable almost equitable partition. Despite the notational complexity, the related computations are quite simple to carry out, as shown in some examples.
\end{abstract}

Key words: Multi-consensus; multi-agent systems; almost equitable partitions; algebraic graph theory.

\section{Introduction}

In this work, devoted to multi-consensus over static networks, we explore the interdependency between the convergence properties of the considered multi-agent system and the structural attributes of the underlying network topology.

In general, consensus in multi-agent systems has attracted a lot of attention due to a wide variety of applications, such as vehicle or robot formation control, swarming, attitude alignment, flocking, rendez-vous problems, and coordinated decision making. Reaching a consensus in a multi-agent system means that the agents' states converge to a common value. After the pioneering works by Vicsek et al. (1995) and Jadbabaie et al. (2003), a thorough theoretical framework about consensus has been given in (Mesbahi and Egerstedt, 2010). The consensus problem has also been widely investigated in more complex systems, such as nonlinear (Moreau, 2005) or high-order systems (Ren and Cao, 2011). Also, over the last years, there has been an increasing interest in the re-

\footnotetext{
* This paper was not presented at any IFAC meeting. Corresponding author L. Ricciardi Celsi. Tel. +39-06-77274034.

Email addresses: salvatore.monaco@uniroma1.it

(Salvatore Monaco),

lorenzo.ricciardicelsi@uniroma1.it (Lorenzo Ricciardi Celsi).
}

design of the network topology under feedback in order to achieve desired consensus, synchronization or gametheoretical equilibrium objectives (Lo Iudice et al., 2015; Barbarossa et al., 2016; Pietrabissa and Ricciardi Celsi, 2018).

Yet, multi-agent systems may sometimes be required to achieve not just a single global consensus value, but different consensuses in different groups, that is, different parts of a multi-agent system are expected to eventually achieve different consensus states simultaneously. Such a situation is defined as multi-consensus (or cluster/group consensus). In general, multi-consensus is currently proving to be more momentous than single consensus in control science, physics, brain science, computer science and economics, with a relevant number of applications, such as space-based interferometers, the design of surveillance and reconnaissance systems, and distributed wireless sensor networks. Hence, it is of great significance to study the behaviour of multiple agents achieving several types of consensus simultaneously. Recently, there have been some interesting investigations in this respect, such as (Blondel et al., 2010), where, in the context of opinion dynamics, the agents are proven to converge to different clusters so that the agents belonging to each cluster share a common opinion. In (Jin and Zheng, 2009), within each group, the agents cooperate and reach a consensus state. However, in that setting, agents in non-independent subsystems actually do 
not reach agreement in the common sense. Yu and Wang (2010) further studied the cluster consensus of multiagent systems in directed networks with time-varying topologies and delays by dividing the network into several corresponding subnetworks. Other interesting works investigating multi-consensus of continuous-time nonlinearly coupled multi-agent systems are (Aeyels and De Smet, 2010) and (Li et al., 2015). Moreover, in (Chen et al., 2011) discrete-time multi-agent systems with a linear protocol and a time-varying topology are discussed. Yet, in the existing literature about the cluster consensus of multi-agent systems, the clusters of agents are formed artificially and the number of clusters is not determined on the basis of the digraph Laplacian matrix.

In particular, this work is aimed at shedding light on the relationship between the number of formed clusters and the structural properties of the digraph Laplacian matrix. Hence, we tackle the problem of multi-consensus, being inspired by the most recent results on the relationship between a specific kind of graph partitions namely, the so-called almost equitable partitions (AEP) - and geometric control theory, especially by the invariance properties associated with such graph partitions (Cardoso et al., 2007; O'Clery et al., 2013; Schaub et al., 2016). Indeed, the almost equitability of a graph partition is an important graph-theoretical property which admits an interesting geometrical interpretation (Martini et al., 2010; Zhang et al., 2014; Monshizadeh et al., 2015; Aguilar and Gharesifard, 2016) and thus can be profitably used to set and solve networked analysis and control problems relying on a geometric approach. In particular, the mathematical foundation for the results presented in this paper has been laid by the seminal work by Caughman and Veerman (2006), where for the first time a block lower triangular structure is given for the Laplacian matrix of digraphs such that algebraic multiplicity of the zero eigenvalue is larger than one.

The purpose of this work is therefore to analyze in detail the multi-consensus problem and relate it to the notion of almost equitable graph partition, by providing a clear formalism for examining how the network topology determines the steady-state behaviour of a multi-agent system.

For the first time, to the best of the authors' knowledge, the consensus values together with the corresponding clusters of network nodes are fully characterized enlightening the role played by the concept of AEP. On the one hand, as the intuition suggests, some of these clusters coincide with the so-called digraph reaches, whose specific consensus values depend each on the initial conditions of the related reach root nodes (see Section 3 for the precise definitions and statements). On the other hand, the remaining clusters, whose characterization is much more complex, identify specific cells belonging to the so-called common part $\mathcal{C}$ of the digraph: the consensus value of each cell is a well-identified linear combination of the consensuses achieved by each reach (see formulae (37) and (38)). These clusters, together with the digraph reaches, define an AEP so that the number of achieved consensuses is equal to the cardinality of the AEP itself.

The paper is organized as follows. Section 2 provides background information and notation. Sections 3 and 4 recall the notions of single consensus, multi-consensus and almost equitable graph partition, thus setting the stage for the subsequent Section 5, which shows the main result relating multi-consensus and almost equitable graph partitions, by (i) providing a necessary and sufficient condition for the global asymptotic stability of multi-consensus and (ii) identifying how many different consensuses can be achieved based on the properties of the digraph Laplacian matrix. Concluding remarks in Section 6 end the paper.

\section{Notation}

Let us consider an unweighted directed graph (or digraph) of order $n$ represented by $\mathcal{G}:=(\mathcal{V}, \mathcal{E})$, where $\mathcal{V}=\left\{w_{1}, \ldots, w_{n}\right\}$ is a finite nonempty node set and $\mathcal{E} \subseteq \mathcal{V} \times \mathcal{V}$ is an edge set of ordered pairs of nodes, called edges. For two distinct nodes $w_{i}, w_{j} \in \mathcal{V}$, we have $\left(w_{i}, w_{j}\right) \in \mathcal{E}$ if there is an edge from $w_{i}$ to $w_{j}$ with $w_{i}$ being the tail and $w_{j}$ being the head of the edge: hence, $w_{i}$ is said to be a neighbour of $w_{j}$. We call any subset $\rho$ of $\mathcal{V}$ a cell of $\mathcal{V}$.

A digraph $\mathcal{G}$ contains a rooted out-branching as a subgraph if it does not contain a directed cycle and if it has a node $w_{\text {root }}$ (i.e., the root node) such that for every other node $w \in \mathcal{V}$ there exists a directed path from $w_{\text {root }}$ to $w$.

We call a collection of cells, given by $\pi=\left\{\rho_{1}, \rho_{2}, \ldots, \rho_{k}\right\}$, a partition of $\mathcal{V}$ if $\rho_{i} \cap \rho_{j}=\emptyset$, whenever $i \neq j$, and $\cup_{i=1}^{k} \rho_{i}=\mathcal{V}$. For a cell $\rho \subseteq \mathcal{V}$, we define the characteristic vector of $\rho$ as $p(\rho) \in \overline{\mathbb{R}}^{n}$ such that:

$$
p_{i}(\rho)= \begin{cases}1 & \text { if } w_{i} \in \rho, \\ 0 & \text { otherwise }\end{cases}
$$

For a partition $\pi=\left\{\rho_{1}, \rho_{2}, \ldots, \rho_{k}\right\}$, we define the $c h a$ racteristic matrix of $\pi$ as $P(\pi)=\left(p\left(\rho_{1}\right) p\left(\rho_{2}\right) \ldots p\left(\rho_{k}\right)\right)$. With $\operatorname{Im} P(\pi)$ we denote the range space of $P(\pi)$, that is, the span of the column vectors of $P(\pi)$. A partition $\pi_{1}$ is said to be finer than another partition $\pi_{2}$, or alternatively $\pi_{2}$ is coarser than $\pi_{1}$, if each cell of $\pi_{1}$ is a subset of some cell of $\pi_{2}$. With the symbol $\rho^{t r}$, we denote a generic trivial cell, i.e., a cell containing one node only.

Moreover, let $\mathbf{1}_{n}$ represent the $n$-dimensional vector of all ones and let the vectors $\mathbf{e}_{1}=\left(\begin{array}{lllll}1 & 0 & 0 & \ldots & 0\end{array}\right)^{T}, \mathbf{e}_{2}=$ $\left(\begin{array}{lllll}0 & 1 & 0 & \ldots & 0\end{array}\right)^{T}, \ldots, \mathbf{e}_{n}=\left(\begin{array}{lllll}0 & 0 & \ldots & 0 & 1\end{array}\right)^{T}$ denote the 
standard basis of $\mathbb{R}^{n}$. Let $G L_{n}(\mathbb{R})$ be the general linear group of all $n \times n$ invertible matrices and let $\mathcal{T}_{n}(\mathbb{R}) \subseteq$ $G L_{n}(\mathbb{R})$ be the set of the $n$ ! permutation matrices $T$. Recall that a permutation matrix is a square matrix obtained from the identity matrix of the same size by a permutation of rows and it is always row-equivalent to the identity matrix. Furthermore, every permutation matrix $T$ is orthogonal, i.e., $T^{T}=T^{-1}$.

Before presenting the main contribution of this paper, some well-known facts are reported for the sake of completeness.

\section{Recalls on Multi-Consensus}

For the purposes of multi-agent system analysis and in order to investigate the related consensus properties, the literature typically refers to the multi-agent system model

$$
\dot{\mathbf{x}}=-L \mathbf{x},
$$

consisting of $n>1$ agents labeled by the node set $\mathcal{V}$, that is, each agent corresponds to a specific node of the underlying digraph $\mathcal{G}$. The state vector is therefore defined as $\mathbf{x}(t):=\operatorname{col}\left(x_{1}(t), \ldots, x_{n}(t)\right)$, with $x_{i}(t)$ denoting the state of node (or agent) $w_{i}$ at time $t$. The matrix $L=$ $\left(l_{i j}\right) \in \mathbb{R}^{n \times n}$, with $(i, j) \in\{1, \ldots, n\} \times\{1, \ldots, n\}$, denotes the in-degree Laplacian matrix of the unweighted digraph $\mathcal{G}$, and the $\lambda_{i}$ 's, for $i \in\{1, \ldots, n\}$, are its eigenvalues.

In particular, we are interested in examining the steadystate behaviour of system (1) in the case when the underlying digraph admits multiple consensuses. A preliminary topological characterization in terms of connectedness is needed in order to specify how multi-consensus situations are reached.

Definition 1 (Mesbahi and Egerstedt, 2010) We distinguish among four different topologies in terms of connectedness.

- A digraph is said to be weakly connected if its disoriented version is connected, that is, if its disoriented version is such that there always exists a path between every pair of nodes and there are no unreachable nodes.

- A digraph is said to be a rooted digraph if it is weakly connected and it contains at least one rooted out-branching.

- A digraph is said to be strongly connected if there always exists a directed path between every pair of nodes and there are no unreachable nodes.

- A digraph is said to be balanced if, for every node, the in-degree (i.e., the number of head ends adjacent to the considered node) and the out-degree (i.e., the number of tail ends adjacent to the considered node) are equal.

Remark 1 A strongly connected digraph is both weakly connected and rooted, but the converse is not necessarily true. Moreover, a balanced digraph is strongly connected, but the converse is not necessarily true.

Definition 1 will be used to specify how the degree of connectedness of the network topology underlying the dynamics (1) impacts on the consensus value that is eventually reached. In this respect, we now give the definition of multi-consensus.

Definition 2 Given a digraph partition $\pi=\left\{\rho_{1}, \rho_{2}, \ldots\right.$, $\left.\rho_{k}\right\}$, multi-consensus is defined as the condition when the differences between the state trajectories of nodes belonging to the same cell of $\pi$ converge to zero as time goes to infinity, i.e.,

$$
\begin{aligned}
& \lim _{t \rightarrow \infty}\left[x_{i}(t)-x_{j}(t)\right]=0, \\
& \forall i, j \mid w_{i} \in \rho_{\beta}, w_{j} \in \rho_{\beta}, i \neq j, \beta=1, \ldots, k .
\end{aligned}
$$

Multi-consensus is equivalent to the asymptotic stability of the following multi-consensus manifold with respect to partition $\pi$ :

$$
\begin{aligned}
& \mathcal{M}^{\pi}=\left\{x \in \mathbb{R}^{n}\left|x_{i}=x_{j}, \forall i, j\right|\right. \\
& \left.w_{i} \in \rho_{\beta}, w_{j} \in \rho_{\beta}, i \neq j, \beta=1, \ldots, k\right\} .
\end{aligned}
$$

As known, classical consensus or multi-consensus is achieved depending on the algebraic multiplicity, $\mu$, of the zero eigenvalue (e.g., see (Yi et al., 2011)).

In particular, if $\mu\left(\lambda_{1}=0\right)>1$, then the multi-agent system (1) converges to a different GAS equilibrium state than classical average consensus. In the following, whenever $\mu\left(\lambda_{1}\right)>1$, we will refer to such a quantity simply as $\mu$.

Proposition 1 Weak connectedness of the digraph $\mathcal{G}$ and the absence of a rooted out-branching in $\mathcal{G}$ are sufficient conditions for the algebraic multiplicity of the zero eigenvalue to grow above 1 , i.e., $\mu>1$, yielding $\operatorname{rank}(L)=n-\mu$. In such a case, the GAS equilibrium state which the multi-agent system (1) converges to is given by

$$
\begin{aligned}
\mathbf{x}_{\infty}^{\prime} & =\lim _{t \rightarrow \infty} \mathbf{x}(t)=\left(\mathbf{u}_{1} \mathbf{v}_{1}\right)^{T} \mathbf{x}_{0}+\left(\mathbf{u}_{2} \mathbf{v}_{2}\right)^{T} \mathbf{x}_{0}+ \\
& +\ldots+\left(\mathbf{u}_{\mu} \mathbf{v}_{\mu}\right)^{T} \mathbf{x}_{0}
\end{aligned}
$$

where $\mathbf{u}_{1}, \ldots, \mathbf{u}_{\mu}$ are $\mu$ distinct and linearly independent eigenvectors associated with the zero eigenvalue of $L$ (i.e., $\left.L \mathbf{u}_{i}=0, i=1, \ldots, k\right)$ such that $\left\{\mathbf{u}_{1}, \ldots, \mathbf{u}_{\mu}\right\}$ is a basis of $\operatorname{ker} L:=\mathcal{U}$.

PROOF. It easily follows from the proof of Theorem 3.12 in (Mesbahi and Egerstedt, 2010), with the only 
difference that in this case we recover (4) since, by assumption, $\lambda_{1}=\lambda_{2}=\ldots=\lambda_{\mu}=0$ and $\lambda_{\mu+1}, \ldots, \lambda_{n}$ are such that their real parts are strictly positive.

Note that the $\mathbf{u}_{i}$ eigenvectors, for $i=1, \ldots, \mu$, are always such that $\sum_{i=1}^{\mu} \mathbf{u}_{i}=\mathbf{1}_{n}$. Note also that the GAS equilibrium condition (4) does not imply that the same value is reached for all the components of the state vector $\mathbf{x}$. In the following Section, we will therefore investigate the properties the network topology has to fulfil in order to allow the agents belonging to suitable clusters of nodes (more precisely, cells) to converge each to the same final value, thus yielding multi-consensus according to Definition 2.

In particular, according to Proposition 1 and Definition 2 , it is possible to numerically compute the distinct consensus values achieved by the agents and, therefore, to identify the different cells $\rho_{i}, i=1, \ldots, \omega$, the agents will form at steady state. Such computations could pave the way for the design of a heuristic procedure that is suitable for calculating the almost equitable partition $\pi_{A E}^{*}$, which will be introduced in Section 5 .

From now till the end of this Section, we will rely on the results appearing in (Caughman and Veerman, 2006) to recall some important properties and introduce our main contribution. In fact, still taking into account the situation when $\mu>1$, there exists a redenomination of nodes yielding a suitable lower-triangularization of the Laplacian matrix, namely allowing to interpret the digraph topology in terms of reaches and thus giving further insight into the kernels of digraph Laplacians.

More in detail, given a Laplacian matrix $L$ associated with the digraph $\mathcal{G}$, we write $w_{j} \rightsquigarrow w_{i}$ if there exists a directed path from node $w_{j}$ to node $w_{i}$. In this respect, for any node $w_{j}$, we define the reachable set from $w_{j}$, $\mathcal{R}\left(w_{j}\right)$, to be the set containing $w_{j}$ and all nodes $w_{i}$ such that $w_{j} \rightsquigarrow w_{i}$. The maximal reachable set from $w_{j}, \mathcal{R}_{\max }\left(w_{j}\right)$, is called a reach (Agaev and Chebotarev, 2005; Caughman and Veerman, 2006). Given the reach $\mathcal{R}_{\max }\left(w_{j}\right)$ from a node $w_{j} \in \mathcal{V}$, then we define $w_{j}$ as the reach root node, that is, the root node for $\mathcal{R}_{\max }\left(w_{j}\right)$, since, by definition of reachable set, $w_{j} \rightsquigarrow w_{i}, \forall w_{i} \in$ $\mathcal{R}_{\max }\left(w_{j}\right)$.

Let $\mathcal{R}_{1}, \ldots, \mathcal{R}_{\mu}$ denote the reaches of $\mathcal{G}$. For each reach $\mathcal{R}_{i}$, we define the exclusive part of $\mathcal{R}_{i}$ to be the set $\mathcal{H}_{i}=$ $\mathcal{R}_{i} \backslash \cup_{j \neq i} \mathcal{R}_{j}$. Likewise, we define the common part of $\mathcal{R}_{i}$ to be the set $\mathcal{C}_{i}=\mathcal{R}_{i} \backslash \mathcal{H}_{i}$. Let $\mathcal{C}=\cup_{i=1}^{\mu} \mathcal{C}_{i}$ denote the union of the common parts of all the reaches.

From Theorem 3.2 in (Caughman and Veerman, 2006), we recall the following statement.

Proposition 2 The algebraic multiplicity of $\lambda_{1}=0$ as an eigenvalue of $L$ equals the number, $\mu$, of reaches of $\mathcal{G}$.
As a result of this, by means of a coordinate change $\overline{\mathbf{x}}=T \mathbf{x}$ that suitably reorders the digraph nodes, $L$ takes the following lower-triangular form:

$$
\bar{L}=\left(\begin{array}{ccccc}
L_{1} & 0_{h_{1} \times h_{2}} & \ldots & 0_{h_{1} \times h_{\mu}} & 0_{h_{1} \times \delta} \\
0_{h_{2} \times h_{1}} & L_{2} & \ldots & 0_{h_{2} \times h_{\mu}} & 0_{h_{2} \times \delta} \\
\vdots & \vdots & \ddots & \vdots & \vdots \\
0_{h_{\mu} \times h_{1}} & 0_{h_{\mu} \times h_{2}} & \ldots & L_{\mu} & 0_{h_{\mu} \times \delta} \\
M_{1} & M_{2} & \ldots & M_{\mu} & M
\end{array}\right),
$$

where the $L_{i}$ 's are $h_{i} \times h_{i}$ Laplacian matrices associated with the $\mathcal{H}_{i}$ 's, the $M_{i}$ 's are $\delta \times h_{i}$ matrices, and $M$ is a square matrix of order $\delta$ associated with the union of the common parts of all the digraph reaches (i.e., with $\mathcal{C})$, with $h_{i}:=\left|\mathcal{H}_{i}\right|$, and $\delta:=|\mathcal{C}|$.

The Laplacian matrix $\bar{L}$, as given in (5), is the result of a coordinate transformation that makes use of permutation matrices and yields a mere reordering of the network nodes.

From the computational point of view, such a permutation can be obtained from the digraph properties outlined above or, equivalently, by calculating the eigenvectors of the Laplacian matrix and by determining a suitable basis for the eigenspace of dimension $\mu$, as further detailed in Section 5. Note that such a basis always exists.

\section{Almost Equitable Partitions}

An important issue in investigating networked dynamical systems is to infer certain network properties from the network topology, which is generally represented by the underlying network digraph. In this respect, some notions from graph theory, in particular the concept of graph partition, have proved rather useful and are reviewed in this Section, since they lay the foundation for the subsequent results reported in Section 5 .

A well-known case of graph partition is the equitable partition, which groups nodes with constant in-degree into cells (Godsil and Royle, 2013). The concept of AEP - also defined as external equitable partition in (O'Clery et al., 2013), or relaxed equitable partition in (Martini et al., 2010) - is less restrictive, demanding that the indegree from nodes in a cell is constant with respect to any other cell but not within each cell.

With respect to the digraph $\mathcal{G}$, for a given cell $\rho \subseteq \mathcal{V}$, we denote the neighbourhood of node $w_{j}$ restricted to cell $\rho$ with

$$
\mathcal{N}\left(w_{j}, \rho\right)=\left\{w_{i} \in \rho:\left(w_{i}, w_{j}\right) \in \mathcal{E}\right\} .
$$


First, we provide a graph-theoretical definition of an AEP.

Definition 3 (Almost Equitable Partition) (Cardoso et al., 200\%; Monshizadeh et al., 2015) A partition $\pi_{A E}=\left\{\rho_{1}, \rho_{2}, \ldots, \rho_{k}\right\}$ is said to be an $A E P$ of $\mathcal{G}$ if, for each $i, j \in\{1,2, \ldots, k\}$, with $i \neq j$, there exists an integer $d_{i j}$ such that $\left|\mathcal{N}\left(w, \rho_{j}\right)\right|=d_{i j}$ for all $w \in \rho_{i}$, where $|\mathcal{N}|$ denotes the cardinality of the set $\mathcal{N}$. In other words, a partition such that each node in $\rho_{i}$ has the same number of neighbours in $\rho_{j}$, for all $i, j$ with $i \neq j$, is an AEP.

The property of almost equitability is equivalent to the invariance of the subspaces generated by the characteristic vectors of its cells. So, we can also give an equivalent definition of an AEP, yet this time provided in terms of invariant subspaces.

Definition 4 (Alternative to Definition 3) A partition $\pi_{A E}$ is said to be an $A E P$ if

$$
\operatorname{LIm} P\left(\pi_{A E}\right) \subset \operatorname{Im} P\left(\pi_{A E}\right) .
$$

Hence, the notion of almost equitability of $\pi_{A E}$ with respect to $\mathcal{G}$ is equivalent to the concept of $L$-invariance of Im $P\left(\pi_{A E}\right)$.

Indeed, by making use of geometric tools, one immediately recovers the quotient graph representation (O'Clery et al., 2013). In particular, $P\left(\pi_{A E}\right)$ is the characteristic matrix of $\pi_{A E}$, which sorts the characteristic vectors of the partition cells by column, according to the definition given in Section 2.

Remark 2 If $\pi_{A E}$ is an $A E P$ of $\mathcal{G}$, then, following the proof of Lemma 7 in (Monshizadeh et al., 2015), we have

$$
L P\left(\pi_{A E}\right)=P\left(\pi_{A E}\right) L_{\pi_{A E}}
$$

where $L_{\pi_{A E}}$ is the Laplacian matrix associated with the quotient graph of $\mathcal{G}$ over $\pi_{A E}$, i.e.,

$$
\left(L_{\pi_{A E}}\right)_{i j}=\left\{\begin{array}{l}
-d_{i j} \quad \text { if } i \neq j \\
s_{i} \text { otherwise }
\end{array}\right.
$$

with $s_{i}=\sum_{j \neq i} d_{i j}$.

Example 1 Consider a digraph with Laplacian matrix

$$
L=\left(\begin{array}{cccc}
-3 & 1 & 1 & 1 \\
0 & 0 & 0 & 0 \\
1 & 1 & -3 & 1 \\
0 & 1 & 0 & -1
\end{array}\right)
$$

By Definition 3, we consider the AEP $\pi_{A E}=\left\{\left\{w_{1}\right.\right.$, $\left.\left.w_{3}\right\}, w_{2}, w_{4}\right\}$, with characteristic matrix

$$
P\left(\pi_{A E}\right)=\left(\begin{array}{lll}
1 & 0 & 0 \\
0 & 1 & 0 \\
1 & 0 & 0 \\
0 & 0 & 1
\end{array}\right)
$$

These matrices satisfy the property $L P\left(\pi_{A E}\right) \subset$ Im $P\left(\pi_{A E}\right)$, thus recovering the L-invariance stated in Definition 4 .

Further insight into this invariance property is provided in (O'Clery et al., 2013). Moreover, the usefulness of such notions is widely documented in the recent literature: e.g., in (Monshizadeh et al., 2015), sufficient conditions in terms of AEPs are derived for diffusively coupled networked systems to be disturbance decoupled, and also conditions for guaranteeing the solvability of the disturbance decoupling problem under feedback are provided.

\section{Main Result Relating Multi-Consensus and Almost Equitable Graph Partitions}

We are now in a position to present the main contribution of the paper, thus outlining and clarifying how the network topology determines some of the properties of the dynamic evolution and of the steady-state behaviour of a multi-agent system in the form (1).

Considering the situation when $\mu>1$, the $L_{i}$ matrices, for $i=1, \ldots, \mu$, in (5) identify each a subgraph $\mathcal{H}_{i}$ of $\mathcal{G}$, for $i=1, \ldots, \mu$, respectively, and such subgraphs $\mathcal{H}_{i}$ are disconnected from each other. Instead, the union of the common parts of all the reaches, $\mathcal{C}$, certainly contains all the leaves in the digraph. Also, note that the assumption of weak connectedness, according to the dynamics $\dot{\overline{\mathbf{x}}}=$ $-\bar{L} \overline{\mathbf{x}}$ with $\bar{L}$ as in (5), implies that each node in $\mathcal{C}$ can be reached starting from any node in each of the $\mathcal{H}_{i}$ 's.

At this point, we present an interesting result, especially concerning the eigenvectors associated with the zero eigenvalue of $L$.

Proposition 3 Given $L$ as the Laplacian of a weakly connected digraph $\mathcal{G}$ with $\mu>1$, after a suitable permutation of the network nodes, such a matrix can be rewritten 


$$
\begin{aligned}
& \bar{L}=T L T^{-1}= \\
& \left(\begin{array}{ccccc}
\left(\begin{array}{cc}
R_{1} & 0 \\
K_{11} & K_{1}
\end{array}\right) & 0_{h_{1} \times h_{2}} & \ldots & 0_{h_{1} \times h_{\mu}} & 0_{h_{1} \times \delta} \\
0_{h_{2} \times h_{1}} & \left(\begin{array}{cc}
R_{2} & 0 \\
K_{12} & K_{2}
\end{array}\right) & \vdots & 0_{h_{2} \times h_{\mu}} & \vdots \\
\vdots & \vdots & \ddots & \vdots & \vdots \\
0_{h_{\mu} \times h_{1}} & 0_{h_{\mu} \times h_{2}} & \vdots & \left(\begin{array}{cc}
R_{\mu} & 0 \\
K_{1 \mu} & K_{\mu}
\end{array}\right) & 0_{h_{\mu} \times \delta} \\
M_{1} & M_{2} & \ldots & M_{\mu} & M
\end{array}\right),
\end{aligned}
$$

where each $\left(\begin{array}{cc}R_{i} & 0 \\ K_{1 i} & K_{i}\end{array}\right)$ block accounts for the corresponding $L_{i}$ block, for $i=1, \ldots, \mu$, in $\bar{L}$ (given as in (5)), $R_{i}$ and $K_{i}$ are square matrices of order $h_{i}-l_{i}$ and $l_{i}$, respectively, with $l_{i}:=|\mathcal{I}|$ and $\mathcal{I}=\mathcal{V} \backslash \mathcal{Q}$, with $\mathcal{Q}$ denoting the subset of the root nodes of the digraph, and also $M$ is the same as in (5) and accounts for the $\mathcal{C}$ set, whose nodes are reachable by any $\mathcal{H}_{i}, i=1, \ldots, \mu$. Moreover, a possible basis for the kernel of $L$, i.e., for $\mathcal{U}$, generated by the $\mathbf{u}_{i}$ 's, takes the form $\left\{\mathbf{u}_{1}, \ldots, \mathbf{u}_{\mu}\right\}$, where

$$
\mathbf{u}_{1}=\left(\begin{array}{c}
\mathbf{1}_{h_{1}} \\
0 \\
\vdots \\
0 \\
\gamma^{1}
\end{array}\right), \mathbf{u}_{2}=\left(\begin{array}{c}
0 \\
\mathbf{1}_{h_{2}} \\
\vdots \\
0 \\
\gamma^{2}
\end{array}\right), \ldots, \mathbf{u}_{\mu}=\left(\begin{array}{c}
0 \\
0 \\
\vdots \\
\mathbf{1}_{h_{\mu}} \\
\gamma^{\mu}
\end{array}\right)
$$

with

$$
\gamma^{i}=\left(\gamma_{w_{1}}^{i} \gamma_{w_{2}}^{i} \ldots \gamma_{w_{\delta}}^{i}\right)^{T}
$$

such that $0<\gamma_{w_{j}}^{i}<1, \forall i \in\{1, \ldots, \mu\}, \forall j \in$ $\{1,2, \ldots, \delta\}$, and $\sum_{i=1}^{\mu} \gamma^{i}=\mathbf{1}_{\delta}$. Moreover, the corresponding left eigenvectors $\mathbf{v}_{i}$, for $i=1, \ldots, \mu$, take the

$$
\begin{aligned}
& \mathbf{v}_{1}=\left(\begin{array}{c}
\left(\begin{array}{c}
\mathbf{v}^{r_{1}} \\
0
\end{array}\right)_{h_{1}} \\
0 \\
\vdots \\
0 \\
0 \\
\vdots \\
0
\end{array}\right), \mathbf{v}^{2}=\left(\begin{array}{c}
0 \\
\left(\mathbf{v}^{r_{2}}\right) \\
0
\end{array}\right){ }_{h_{2}}\left(\begin{array}{c} 
\\
\vdots \\
0 \\
0 \\
\vdots \\
0
\end{array}\right), \ldots, \\
& \ldots, \mathbf{v}^{\mu}=\left(\begin{array}{c}
0 \\
0 \\
\vdots \\
\left(\mathbf{v}^{r_{m}}\right. \\
0)_{h_{\mu}} \\
0 \\
\vdots \\
0
\end{array}\right)
\end{aligned}
$$

where $r_{i}:=\left|\mathcal{Q}_{i}\right| \leq h_{i}$, with $\mathcal{Q}_{i}$ denoting the subset of the roots of the subgraph $\mathcal{H}_{i}$, for $i=1, \ldots, \mu$.

PROOF. For each $L_{i}$, if $\mathcal{I} \neq 0$, there always exists a suitable permutation matrix $T$ such that $\bar{L}_{i}=T L_{i} T^{-1}=\left(\begin{array}{cc}R_{i} & 0 \\ K_{1 i} & K_{i}\end{array}\right)$ holds. By applying this property to each $L_{i}$ block, for $i=1, \ldots, \mu$, given as in (5), we obtain (12). For the rest of the proof, refer to Theorem 3.2 in (Caughman and Veerman, 2006).

Note that each $\mathbf{u}_{i}$ is associated with each $\mathcal{H}_{i}$, while the vector $\gamma^{i}$ in each $\mathbf{u}_{i}$ is associated with $\mathcal{C}$, i.e., with the union of the common parts of all the reaches, and hence must satisfy the relation $\sum_{i=1}^{\mu} \gamma^{i}=\mathbf{1}_{\delta}$.

Moreover, in the $\overline{\mathbf{x}}=T \cdot \mathbf{x}$ coordinates, the left eigenvectors take the form (15) and, specifically, the $v_{i}^{r_{z}}$ 's, for $i=1, \ldots, r_{z}$ and $z=1, \ldots, \mu$, are such that $v_{i}^{r_{z}} \neq 0$ and $\sum_{i=1}^{\mu} v_{i}^{r_{z}}=1$.

The permutation leading to (12) stems not just from (Caughman and Veerman, 2006), but also from the studies in distributed coordination about a graphical decomposition criterion for showing the convergence of infinite products of stochastic matrices (Chen et al., 2016) as 
well as from the recent results about symmetry-based necessary conditions for controllability of multi-agent networked control systems (Aguilar and Gharesifard, 2016). Yet, to the best of the authors' knowledge, for the case when $\mu>1$, no explicit transformed representation of the Laplacian matrix with main diagonal blocks accounting for the $\mathcal{H}_{i}$ 's (such as the one in (12)) has been given before.

Moreover, the $\mu$ reaches identify as many subgraphs $\mathcal{H}_{i}, i=1, \ldots, \mu$, which can be distinguished into separate cells of a suitable AEP - namely, of $\pi_{A E}^{\prime}$ as proven by Proposition 4 - since, according to the lower-triangular representation (12), there is no mutual connection among the $\mathcal{H}_{i}$ 's.

Example 2 Consider a directed graph $\mathcal{G}$ with Laplacian matrix

$$
L=\left(\begin{array}{cccccc}
1 & 0 & -1 & 0 & 0 & 0 \\
0 & 1 & 0 & 0 & -1 & 0 \\
0 & 0 & 1 & 0 & 0 & -1 \\
-1 & -1 & 0 & 2 & 0 & 0 \\
0 & -1 & 0 & 0 & 1 & 0 \\
0 & 0 & -1 & 0 & 0 & 1
\end{array}\right)
$$

Let us change coordinates according to the permutation matrix

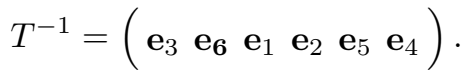

Then, we obtain the transformed Laplacian matrix

$$
\begin{aligned}
\bar{L} & =T L T^{-1}= \\
& =\left(\begin{array}{ccc|cc|c}
1 & -1 & 0 & 0 & 0 & 0 \\
-1 & 1 & 0 & 0 & 0 & 0 \\
0 & -1 & 1 & 0 & 0 & 0 \\
\hline 0 & 0 & 0 & 1 & -1 & 0 \\
0 & 0 & 0 & -1 & 1 & 0 \\
\hline-1 & 0 & 0 & -1 & 0 & 2
\end{array}\right),
\end{aligned}
$$

with

$$
\begin{aligned}
& L_{1}=\left(\begin{array}{ccc}
1 & -1 & 0 \\
-1 & 1 & 0 \\
0 & -1 & 1
\end{array}\right), \\
& L_{2}=\left(\begin{array}{cc}
1 & -1 \\
-1 & 1
\end{array}\right),
\end{aligned}
$$

and where, in the original $\mathbf{x}$-coordinates, $w_{3}$ and $w_{6}$ are the reach root nodes of $\mathcal{H}_{1}$, and $w_{2}$ and $w_{5}$ are the reach root nodes of $\mathcal{H}_{2}$.
Proposition 3 can be interpreted as an extension of the related result in (Caughman and Veerman, 2006) concerning the left and right eigenvectors associated with the Laplacian matrix of a weakly connected digraph with $\mu>1$.

As a by-product of Proposition 3, that is, when the Laplacian can be turned into the form (12), we can state the following result.

Proposition 4 A partition $\pi_{A E}^{\prime}=\left\{\left\{\mathcal{H}_{1}\right\},\left\{\mathcal{H}_{2}\right\}, \ldots\right.$, $\left.\left\{\mathcal{H}_{\mu}\right\}, \rho_{\mu+1}^{t r}, \rho_{\mu+2}^{t r}, \ldots, \rho_{\mu+\delta}^{t r}\right\}$ with characteristic matrix in the $\overline{\mathbf{x}}$-coordinates

$$
P\left(\pi_{A E}^{\prime}\right)=\left(\begin{array}{ccccc}
\mathbf{1}_{h_{1}} & 0_{h_{1} \times 1} & \ldots & 0_{h_{1} \times 1} & 0_{h_{1} \times \delta} \\
0_{h_{2} \times 1} & \mathbf{1}_{h_{2}} & \ldots & 0_{h_{2} \times 1} & 0_{h_{2} \times \delta} \\
\vdots & \vdots & \vdots & \vdots & \vdots \\
0_{h_{\mu} \times 1} & 0_{h_{\mu} \times 1} & \ldots & \mathbf{1}_{h_{\mu}} & 0_{h_{\mu} \times \delta} \\
- & - & - & - & - \\
0_{\delta \times 1} & 0_{\delta \times 1} & \ldots & 0_{\delta \times 1} & I_{\delta},
\end{array}\right)
$$

i.e., such that it collects all the nodes belonging to each $\mathcal{H}_{i}$ into a separate cell and the rest of the digraph nodes each into a trivial cell $\rho_{\mu+j}^{t r}$ for $j=1, \ldots, \delta$, with $I_{\delta}$ denoting the identity matrix of order $\delta$, is an AEP of a digraph $\mathcal{G}$ whose Laplacian is such that $\mu>1$.

PROOF. Since

$$
\bar{L} P\left(\pi_{A E}\right)=\left(\begin{array}{ccccc}
0 & 0 & \ldots & 0 & 0 \\
M_{1} & M_{2} & \ldots & M_{\mu} & M
\end{array}\right)
$$

with $P\left(\pi_{A E}\right)$ as in $(20)$, then

$$
\begin{array}{r}
\bar{L} P\left(\pi_{A E}\right) \subset \operatorname{span}\left(\begin{array}{c}
0_{\left.\left(h_{1}+\ldots+h_{\mu}\right) \times \delta\right)} \\
I_{\delta}
\end{array}\right)= \\
=\operatorname{span}\left\{\left(\begin{array}{c}
0 \\
\mathbf{e}_{1}
\end{array}\right), \ldots,\left(\begin{array}{c}
0 \\
\mathbf{e}_{\delta}
\end{array}\right)\right\},
\end{array}
$$

with $\mathbf{e}_{1}, \ldots \mathbf{e}_{\delta}$ denoting the standard basis of $\mathbb{R}^{\delta}$.

With this in mind, we can show the interesting result proposed by Theorem 1. But, first, we need to outline a particular condition, related to AEPs, that will be instrumental in proving the subsequent Theorem 1 . This is aimed at determining an AEP, denoted with $\pi_{A E}^{*}$, that is coarser than $\pi_{A E}^{\prime}$ and such that its $k<\delta$ cells in $\mathcal{C}$ are as many as the groups of identical components in the $\gamma^{i}$ vectors, $i=1, \ldots, \mu$. Indeed, such $k$ cells in $\mathcal{C}$ can be 
proved to identify the different clusters the considered multi-agent system is divided into at steady state.

Remark 3 A necessary external condition for a subset of nodes in $\mathcal{C}$ to compose a cell of an AEP that is coarser than $\pi_{A E}^{\prime}$ (only with respect to the trivial cells $\rho_{\mu+j}^{t r}, j=$ $1, \ldots, \delta$, accounting for the nodes in $\mathcal{C})$ is that they have the same in-degree with respect to each $\mathcal{H}_{i}, i=1, \ldots, \mu$. This reflects into a property of the row sums of each $M_{i}$ matrix in (12), for $i=1, \ldots, \mu$ : more precisely, in the $\delta$-dimensional vector $M_{i} \mathbf{1}_{h_{i}}$, the components that are associated with nodes belonging to the same cell $\rho_{j}, j=$ $1, \ldots, k$, must be equal.

Theorem 1 A necessary and sufficient condition for a subset of nodes in $\mathcal{C}$ to belong to the same cell $\rho$ of an $A E P$ is that the components of the $\gamma^{i}$ vectors, $i=1, \ldots, \mu$, associated with such nodes are equal.

PROOF. For the sake of clarity, let the nodes in $\mathcal{C}$ be denoted with $w_{1}, \ldots, w_{\delta}$ and let $\rho_{1}, \ldots, \rho_{k}$ denote $k$ cells - each with cardinality $\zeta_{i}:=\left|\rho_{i}\right| \geq 0(i=1, \ldots, k)$ so that $\sum_{i=1}^{k} \zeta_{i}=\delta-$ partitioning $\mathcal{C}$ in the following way:

$$
\left\{\begin{array}{l}
\rho_{1}=\left\{w_{1}, \ldots, w_{\zeta_{1}}\right\}, \\
\rho_{2}:=\left\{w_{\zeta_{1}+1}, \ldots, w_{\zeta_{1}+\zeta_{2}}\right\}, \\
\vdots \\
\rho_{k}:=\left\{w_{\zeta_{1}+\ldots+\zeta_{k-1}+1}, \ldots, w_{\delta}\right\} .
\end{array}\right.
$$

Furthermore, the condition that, for each cell $\rho_{j}, j=$ $1, \ldots, k$, the elements of the $\gamma^{i}$ vectors, $i=1, \ldots, \mu$, associated with the nodes belonging to cell $\rho_{j}$ be equal can be formalized as follows:

$$
\begin{aligned}
& \begin{cases}\gamma_{w_{1}}^{i} & =\ldots=\gamma_{w_{\zeta_{1}}}^{i}:=\tau_{1}^{i}, \\
\gamma_{w_{\zeta_{1}+1}}^{i} & =\ldots=\gamma_{w_{\zeta_{1}+\zeta_{2}}}^{i}:=\tau_{2}^{i}, \\
& \vdots \\
\gamma_{w_{\zeta_{1}+\ldots \zeta_{k-1}+1}}^{i} & =\ldots=\gamma_{w_{\delta}}^{i}:=\tau_{k}^{i},\end{cases} \\
& \forall i \in\{1,2, \ldots, \mu\},
\end{aligned}
$$

where $\gamma_{w_{j}}^{i}$ denotes the element of the $\gamma^{i}$ vector that is associated with node $w_{j}$ in $\mathcal{C}$ according to (23), and $\tau_{j}^{i} \in$ $\mathbb{R}$, for $j=1, \ldots, k, i=1, \ldots, \mu$.

By means of a suitable coordinate change, in the form $\tilde{\mathbf{x}}=T \mathbf{x}$, with respect to the nodes in $\mathcal{C}$ only - which are denoted by $\mathbf{x}_{\delta}$ in the $\mathbf{x}=\left(\begin{array}{llll}\mathbf{x}_{1} & \mathbf{x}_{2} & \ldots & \mathbf{x}_{\delta}\end{array}\right)$-coordinates - we reorder the nodes in $\mathcal{C}$, identified in the new $\tilde{\mathbf{x}}$ - coordinates by $\tilde{\mathbf{x}}_{\delta}$, so that

$$
\tilde{\mathbf{x}}_{\delta}=T_{\delta} \mathbf{x}_{\delta}=\left(\begin{array}{c}
x_{w_{1}} \\
\vdots \\
x_{w_{\zeta_{1}}} \\
x_{w_{\zeta_{1}+1}} \\
\vdots \\
x_{w_{\zeta_{1}+\zeta_{2}}} \\
\vdots \\
x_{w_{\zeta_{1}+\ldots+\zeta_{k-1}}} \\
\vdots \\
x_{w_{\delta}}
\end{array}\right) .
$$

We now have that the system dynamics is expressed by $\dot{\tilde{\mathbf{x}}}=-\tilde{L} \tilde{\mathbf{x}}$, where

$$
\tilde{L}=\left(\begin{array}{ccccc}
L_{1} & 0 & \cdots & 0 & 0 \\
0 & L_{2} & \cdots & 0 & 0 \\
\vdots & \vdots & \ddots & \vdots & \vdots \\
0 & 0 & \cdots & L_{\mu} & 0 \\
\tilde{M}_{1} & \tilde{M}_{2} & \cdots & \tilde{M}_{\mu} & \tilde{M}
\end{array}\right)
$$

whose only difference from $\bar{L}$ is due to the reordering of the nodes belonging to $\mathcal{C}$.

(Necessity) We need to prove that, if the cells $\rho_{1}, \ldots, \rho_{k}$ form an AEP among the nodes of the $\mathcal{C}$ set according to (23), then, for each cell $\rho_{j}, j=1, \ldots, k$, the elements of the $\gamma^{i}$ vectors, $i=1, \ldots, \mu$, associated with the nodes belonging to cell $\rho_{j}$ are equal, i.e., (24) holds.

By assumption, for each cell $\rho_{j}, j=1, \ldots, k$, the sum of the columns of $\tilde{M}$ associated with the nodes belonging to cell $\rho_{j}$ is a linear combination of the characteristic vectors of the cells $\rho_{j}, j=1, \ldots, k$, which the $\mathcal{C}$ set is assumed to be partitioned into, i.e.,

$$
\tilde{M} p\left(\rho_{j}\right)=\sum_{\eta=1}^{k} \varepsilon_{\eta}^{j} p\left(\rho_{\eta}\right), \quad j=1, \ldots, k .
$$

In particular, condition (27) is equivalent to saying that each node belonging to any cell $\rho_{j}, j=1, \ldots, k$, has the same in-degree evaluated with respect to any other cell $\rho_{\theta}$, for $\theta \in\{1, \ldots, k\} \backslash\{j\}$. Moreover, since $\rho_{1}, \ldots, \rho_{k}$ are cells forming an AEP of $\mathcal{C}$ by assumption, then the matrix product $\tilde{M} p\left(\rho_{j}\right), j=1, \ldots, k$, is invariant with respect to any further addition of edges inside such cells. 
On the other hand, since

$$
\tilde{\mathbf{u}}_{1}=\left(\begin{array}{llll}
\mathbf{1}_{h_{1}} & 0 \ldots 0 & \tilde{\gamma}^{1}
\end{array}\right)^{T}, \ldots, \tilde{\mathbf{u}}_{\mu}=\left(\begin{array}{lllll}
0 & 0 & \ldots & \mathbf{1}_{h_{\mu}} & \tilde{\gamma}^{\mu}
\end{array}\right)^{T}
$$

are right eigenvectors of $\tilde{L}$ in $(26)$, it follows that

$$
\tilde{M}_{i} \mathbf{1}_{h_{i}}+\tilde{M} \tilde{\gamma}^{i}=0, \quad i=1, \ldots, \mu .
$$

With this in mind, $\tilde{M}$ can be rewritten in the following form:

$$
\tilde{M}=\left(\Gamma_{j z}\right)_{k \times k}
$$

where each $\Gamma_{j z}$ block is a $\zeta_{j} \times \zeta_{z}$ matrix.

In particular, the sum of the columns of the main diagonal blocks $\Gamma_{j j}$, each being a nonsingular square matrix of order $\zeta_{j}$, for $j=1, \ldots, k$, yields a vector with as many components as the cardinality of cell $\rho_{j}, j=1, \ldots, k$. Moreover, such a sum is a vector whose components are all equal to a positive integer constant, i.e.,

$$
\Gamma_{j j} \mathbf{1}_{\zeta_{j}}=\sigma \mathbf{1}_{\zeta_{j}}, \quad \sigma \in \mathbb{Z}^{+}, j=1, \ldots, k
$$

Note also that each off-diagonal block $\Gamma_{j z}, j=$ $1, \ldots, k, z=1, \ldots, k, z \neq j$, is such that its row sums are all equal to a nonnegative integer constant, i.e.,

$$
\Gamma_{j z} \mathbf{1}_{\zeta_{z}}=\phi \mathbf{1}_{\zeta_{z}}, \quad \phi \in \mathbb{N}
$$

In plain words, equations (27) and (31) remain invariant with respect to any change in the position, along the rows, of the ones appearing in the off-diagonal blocks of $\tilde{M}$.

Hence, (27), (29), (31), and (32), relying on Remark 3, imply that:

$$
\begin{aligned}
& \tilde{M} \tilde{\gamma}^{i}=\left(\Gamma_{j z}\right)_{k \times k}\left(\begin{array}{c}
\tilde{\gamma}_{1}^{i} \\
\vdots \\
\tilde{\gamma}_{k}^{i}
\end{array}\right)= \\
& =\sum_{j=1}^{k} \tau_{j}^{i} p\left(\rho_{j}\right)=\tau_{1}^{i}\left(\begin{array}{c}
\mathbf{1}_{\zeta_{1}} \\
0_{\zeta_{2}} \\
\vdots \\
0_{\zeta_{k}}
\end{array}\right)+\tau_{2}^{i}\left(\begin{array}{c}
0_{\zeta_{1}} \\
\mathbf{1}_{\zeta_{2}} \\
\vdots \\
0_{\zeta_{k}}
\end{array}\right)+ \\
& +\ldots+\tau_{k}^{i}\left(\begin{array}{c}
0_{\zeta_{1}} \\
0_{\zeta_{2}} \\
\vdots \\
\mathbf{1}_{\zeta_{k}}
\end{array}\right), \quad i=1, \ldots, \mu
\end{aligned}
$$

where, for each $i, 0_{\zeta_{i}}$ is a vector whose $\zeta_{i}$ components are all zeros. This allows to recover the thesis, i.e., that the solution $\tilde{\gamma}^{i}$ to (33) is such that $\gamma^{i}=T_{\delta}^{-1} \tilde{\gamma}^{i}$, with $\tilde{\gamma}_{j}^{i}=\tau_{j}^{i} \mathbf{1}_{\zeta_{j}}$, for $i=1, \ldots, \mu$, and $j=1, \ldots, k$, yields $(24)$ for a partition of $\mathcal{C}$ defined as in (23).

(Sufficiency) We now need to prove that, if, for each cell $\rho_{j}, j=1, \ldots, k$, the elements of the $\gamma^{i}$ vectors, $i=$ $1, \ldots, \mu$, associated with the nodes belonging to cell $\rho_{j}$ are equal (as in (24)), then the cells $\rho_{1}, \ldots, \rho_{k}$ form an AEP among the nodes of the $\mathcal{C}$ set as in (23).

First of all, let $\rho_{1}, \ldots, \rho_{k}$ denote a generic partition of $\mathcal{C}$. The assumption that, for each cell $\rho_{j}, j=1, \ldots, k$, the elements of the $\gamma^{i}$ vectors, $i=1, \ldots, \mu$, associated with the nodes belonging to cell $\rho_{j}$ are equal can be formalized as follows:

$$
\gamma^{i}=\sum_{j=1}^{k} \tau_{j}^{i} p\left(\rho_{j}\right), \quad i=1, \ldots, \mu
$$

with $\tau_{j}^{i} \in \mathbb{R}$, for $i=1, \ldots, \mu, j=1, \ldots, k$.

So, we need to prove that (29) and (34) imply that $\rho_{1}, \ldots, \rho_{k}$ actually form an AEP of $\mathcal{C}$. Indeed, if, after a suitable coordinate change $\tilde{\mathbf{x}}=T \mathbf{x}$ satisfying (25), we substitute (34) into (29), we get:

$$
\begin{aligned}
& \tilde{M}_{i} \mathbf{1}_{h_{i}}+\left(\begin{array}{cccc}
\Gamma_{11} & \Gamma_{12} & \ldots & \Gamma_{1 k} \\
\Gamma_{21} & \Gamma_{22} & \ldots & \Gamma_{2 k} \\
\vdots & \vdots & \ddots & \vdots \\
\Gamma_{k 1} & \Gamma_{k 2} & \ldots & \Gamma_{k k}
\end{array}\right) \cdot T_{\delta} \cdot\left(\sum_{j=1}^{k} \tau_{j}^{i} p\left(\rho_{j}\right)\right)=0 \\
& i=1, \ldots, \mu
\end{aligned}
$$

Such a relation holds only if, in the $\delta$-dimensional vector $\tilde{M}_{i} \mathbf{1}_{h_{i}}$, the components that are associated with nodes belonging to the same cell $\rho_{j}$ are equal, for each cell $\rho_{j}, j=1, \ldots, k$. Since, by Remark 3 , this is a necessary condition for $\rho_{1}, \ldots, \rho_{k}$ to form an AEP of $\mathcal{C}$, then $(29)$ and (34) imply (27), thus recovering the thesis required for the desired sufficiency proof.

From Theorem 1, it follows that the characteristic vectors of the cells of a suitable AEP denoted with $\pi_{A E}^{*}$ (with respect to which $\pi_{A E}^{\prime}$ is finer) identify each a different set of nodes whose states converge to the same steady-state value. More precisely, according to the lower-triangular structure (5), the cells of $\pi_{A E}^{*}$ are identified by the $\mathcal{H}_{i}$ 's, on the one hand, and by a suitable sub-partition $\rho_{1}, \ldots, \rho_{k}$ of $\mathcal{C}$ (yielding the relevant property outlined by Theorem 1), on the other hand. 
Corollary 1 The partition $\pi_{A E}^{*}:=\left\{\left\{\mathcal{H}_{1}\right\},\left\{\mathcal{H}_{2}\right\}, \ldots\right.$, $\left.\left\{\mathcal{H}_{k}\right\}, \rho_{\mu+1}, \rho_{\mu+2}, \ldots, \rho_{\mu+k}\right\}$, with $k<\delta$, defined so that each cell $\rho_{\mu+j}$, for $j=1, \ldots, k$, groups the nodes in $\mathcal{C}$ associated with equal components of the $\gamma^{i}$ vectors (for $i=1, \ldots, \mu)$, is the coarsest $A E P$ of $\mathcal{G}$.

PROOF. The proof follows from Theorem 1 and from the fact that a node appearing in any of the $\mathcal{H}_{i}$ 's and a node appearing in $\mathcal{C}$ cannot belong to the same cell of an AEP.

We can now state the final result linking multi-consensus to the notion of AEP.

Corollary 2 A multi-agent system in the form (1), characterized by an underlying weakly connected digraph $\mathcal{G}$ with Laplacian matrix such that $\mu>1$, achieves multi-consensus with respect to groups of nodes which coincide with the cells of partition $\pi_{A E}^{*}$ : namely, for each $i=1, \ldots, \mu$, the nodes belonging to $\mathcal{H}_{i}$ converge to a value which depends on the initial conditions of the reach root nodes of $\mathcal{H}_{i}$, whereas, for each $j=1, \ldots, k$, the nodes belonging to cell $\rho_{\mu+j}$ in $\mathcal{C}$ converge to a distinct convex combination of the consensuses achieved within the $\mathcal{H}_{i}$ 's $(i=1, \ldots, \mu)$.

PROOF. The proof follows from Proposition 1 and Theorem 1. Indeed, let us apply a coordinate change $\overline{\mathbf{x}}=T \mathbf{x}=\left(\overline{\mathbf{x}}_{1} \ldots \overline{\mathbf{x}}_{i} \ldots \overline{\mathbf{x}}_{\mu} \overline{\mathbf{x}}_{\delta}\right)^{T}$ satisfying (25), where each $\overline{\mathbf{x}}_{i}$ groups the nodes belonging to $\mathcal{H}_{i}$, for $i=1, \ldots, \mu$, and $\overline{\mathbf{x}}_{\delta}$ groups all the nodes belonging to $\mathcal{C}$. Let the vector of the initial conditions of the transformed system be denoted with $\overline{\mathbf{x}}_{0}$ and composed internally as follows:

$$
\begin{aligned}
& \overline{\mathbf{x}}_{0}=\left(\begin{array}{lllll}
\left(\overline{\mathbf{x}}_{0}^{r_{1}}\right)^{T} & 0 & \ldots & 0
\end{array}\right)_{h_{1}}\left(\begin{array}{lllll}
\left(\overline{\mathbf{x}}_{0}^{r_{2}}\right)^{T} & 0 & \ldots & 0
\end{array}\right)_{h_{2}} \ldots \\
& \left.\ldots\left(\begin{array}{lllll}
\left(\overline{\mathbf{x}}_{0}^{r_{\mu}}\right)^{T} & 0 & \ldots & 0
\end{array}\right)_{h_{\mu}}\right)^{T},
\end{aligned}
$$

where, consistently with the chosen coordinate change that satisfies (25), each $\overline{\mathbf{x}}_{0}^{r_{z}}$ vector collects the initial conditions of the reach root nodes of $\mathcal{H}_{z}$ only (i.e., of the nodes belonging to $\mathcal{Q}_{z}$ ), for $z=1, \ldots, \mu$.

Then, according to Proposition 1, the state of the transformed multi-agent system $\dot{\overline{\mathbf{x}}}=-\bar{L} \overline{\mathbf{x}}$, with $\bar{L}$ as in (12), converges to a GAS equilibrium state

$$
\overline{\mathbf{x}}_{\infty}^{\prime}=\left(\begin{array}{c}
\mathbf{1}_{h_{1}} \\
0 \\
\vdots \\
0 \\
\tau_{1}^{1} \mathbf{1}_{\zeta_{1}} \\
\vdots \\
\tau_{k}^{1} \mathbf{1}_{\zeta_{k}}
\end{array}\right) \sum_{i=1}^{r_{1}} v_{i}^{r_{1}} \cdot \bar{x}_{0, i}^{r_{1}}+\left(\begin{array}{c}
0 \\
\mathbf{1}_{h_{2}} \\
\vdots \\
0 \\
\tau_{1}^{2} \mathbf{1}_{\zeta_{1}} \\
\vdots \\
\tau_{k}^{2} \mathbf{1}_{\zeta_{k}}
\end{array}\right) \sum_{i=1}^{r_{2}} v_{i}^{r_{2}} \cdot \bar{x}_{0, i}^{r_{2}+}
$$$$
+\ldots+\left(\begin{array}{c}
0 \\
0 \\
\vdots \\
\mathbf{1}_{h_{\mu}} \\
\tau_{1}^{\mu} \mathbf{1}_{\zeta_{1}} \\
\vdots \\
\tau_{k}^{\mu} \mathbf{1}_{\zeta_{k}}
\end{array}\right) \sum_{i=1}^{r_{\mu}} v_{i}^{r_{\mu}} \cdot \bar{x}_{0, i}^{r_{\mu}}=
$$

$$
=\left(\begin{array}{c}
\mathbf{1}_{h_{1}} \cdot c^{1} \\
\mathbf{1}_{h_{2}} \cdot c^{2} \\
\vdots \\
\mathbf{1}_{h_{\mu}} \cdot c^{\mu} \\
\mathbf{1}_{\zeta_{1}} \cdot \sum_{z=1}^{\mu} \tau_{1}^{z} c^{z} \\
\vdots \\
\mathbf{1}_{\zeta_{k}} \cdot \sum_{z=1}^{\mu} \tau_{k}^{z} c^{z}
\end{array}\right)
$$

where $\bar{x}_{0, i}^{r_{z}}$ is the $i$-th component of the $\overline{\mathbf{x}}_{0}^{r_{z}}$ vector in (36), and the scalars $c^{1}, \ldots, c^{\mu}$ are such that

$$
c^{z}=\sum_{m=1}^{r_{z}} v_{m}^{r_{z}} \cdot \bar{x}_{0, \xi}^{r_{z}},
$$

for $z=1, \ldots, \mu$, with $\tau_{i}^{j} \in \mathbb{R}$ for $i=1, \ldots, \zeta_{j}$ and $j=1, \ldots, k$, and $v_{m}^{r_{z}}$ denoting the $m$-th component of the $\mathbf{v}^{r_{z}}$ vector introduced in (15), for $m=1, \ldots, r_{z}$. Note that the extrema of the sums are chosen so as to consider the initial conditions of the nodes belonging to $\mathcal{Q}_{i}$ only $(i=1, \ldots, \mu)$.

As a result, the $\gamma_{i}$ vectors in (34), for $i=1, \ldots, \mu$, can be written as

$$
\gamma^{i}=\mathbf{1}_{\zeta_{i}} \sum_{z=1}^{\mu} \tau_{i}^{z} c^{z}
$$

with $c^{z}$ as in (38), denoting the contribution of the reach root nodes of order $z$ to the $i$-th consensus value in $\mathcal{C}$. 


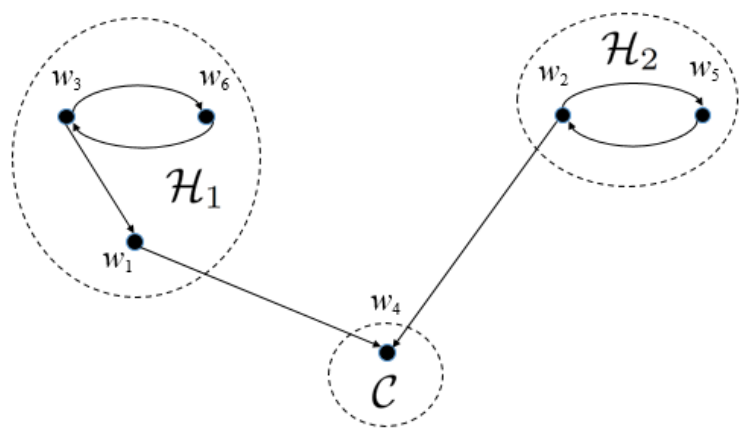

Fig. 1. Representation of $\pi_{A E}^{*}$ for Example 2. The three cells achieve as many distinct consensuses.

Remark 4 The $\gamma^{i}$ vectors, for $i=1, \ldots, \mu$, appearing in (39) have $\delta$ components which can be divided into groups of $k<\delta$ equal components (i.e., as many as the cells of $\pi_{A E}^{*}$ in $\mathcal{C}$ ) as a consequence of Theorem 1. Hence, a system in the form (1), with underlying digraph $\mathcal{G}$ such that $\mu>1$, yields $\mu+k$ distinct consensuses, satisfying, according to Definition 2,

$$
\mathbf{x} \rightarrow \mathcal{M}^{\pi_{A E}^{*}}
$$

Such a number of distinct consensuses is equal to the cardinality of $\pi_{A E}^{*}$.

This implies that a group of nodes in $\mathcal{C}$, belonging to the same cell of $\pi_{A E}^{*}$, converge to the same steady-state value. Such a situation at steady state is clearly a multiconsensus one.

As regards Example 2, the steady state of the related multi-agent system satisfies Theorem 1, Definition 1 and Corollary 2 with $\mu=2$ and $k=1$ - i.e., $\pi_{A E}^{*}=\left\{\left\{\mathcal{H}_{1}\right\},\left\{\mathcal{H}_{2}\right\}, \rho_{3}\right\}$. Hence, it yields 3 distinct consensuses: one for nodes $w_{1}, w_{3}$ and $w_{6}$ composing $\mathcal{H}_{1}$, one for nodes $w_{2}$ and $w_{5}$ composing $\mathcal{H}_{2}$, and one for node $w_{4}$ composing $\rho_{3} \equiv\{\mathcal{C}\}$, according to the transformed Laplacian matrix (18).

In general, $\mathcal{C}$ contains several nodes, and, in this respect, the proof of Corollary 2 (namely, formulae (37) and (38)) highlights that the nodes in $\mathcal{C}$ belonging to the same cell $\rho_{\mu+j}(j=1, \ldots, k)$ of $\pi_{A E}^{*}$ converge to the same convex combination of the consensuses achieved within the $\mathcal{H}_{i}$ 's $(i=1, \ldots, \mu)$.

It is interesting to note that the AEP $\pi_{A E}^{*}$ can be computed by making use of the algorithm proposed in Section IV of (Zhang et al., 2014). Indeed, Zhang et al., with respect to the multi-agent system dynamics

$$
\dot{\mathbf{x}}=-L \mathbf{x}+M \mathbf{u},
$$

propose an algorithm for computing the coarsest AEP such that the range space of its characteristic matrix contains $\operatorname{Im} M$.

Remark 5 The algorithm proposed in Section IV of (Zhang et al., 2014) allows to compute $\pi_{A E}^{*}$, as introduced in Corollary 1, with respect to the multi-agent system dynamics (41), if the input matrix $M$ is chosen so that its columns identify each a separate reach in the digraph - i.e., for each digraph reach, each entry of the related column of $M$ is 1 if the corresponding node belongs to the considered reach, and 0 otherwise.

This can be easily verified on Example 2. Remark 5 also implies that the algorithm proposed in Section IV of (Zhang et al., 2014), which refers only to connected undirected graphs whose Laplacian matrix is such that $\mu\left(\lambda_{1}\right)=1$, can be successfully extended to the case of a rooted digraph whose Laplacian matrix is such that $\mu\left(\lambda_{1}\right)>1$.

\section{Conclusion}

In this paper, the multi-consensus problem in multiagent systems has been investigated by putting the Laplacian matrix of the underlying digraph in a form which specializes the one proposed by Caughman and Veerman (2006) and thus gives insight into the topological structure of the networked system itself. On this basis, it is shown that multi-consensus is achieved when the underlying digraph admits a suitable almost equitable partition denoted with $\pi_{A E}^{*}$. In particular, according to the definitions given above, on the one hand, a consensus is achieved for the states of all the nodes belonging to the exclusive part of each digraph reach, while, on the other hand, different consensuses are achieved by the states of the nodes belonging to the union of the common parts of all the reaches, each of these consensuses being a convex combination of the influences of the exclusive reaches. Thus, the total number of distinct consensuses achieved by the multi-agent system is equal to the number of cells of partition $\pi_{A E}^{*}$. By contrast with the existing literature, in this paper the relationship between the number of cells and the Laplacian is outlined, and the values of the distinct consensuses are precisely specified.

The authors are currently drawing up techniques for efficiently computing the eigenvectors associated with the zero eigenvalue of the digraph Laplacian according to the structure introduced in Proposition 3, especially in the context of complex networks - namely, networks with an extremely large number of agents, with large algebraic multiplicity of the zero eigenvalue of the Laplacian matrix, and yielding a large number of distinct consensuses. Also, the authors are drawing up an algorithm to permutate the nodes of the Laplacian matrix of a weakly connected digraph in order to generate the lower-triangular form (12). 
Finally, further study will be focused on multiconsensusability, on the one hand, when the single agents evolve in $n$-dimensional spaces $(n>1)$ and, on the other hand, in multi-agent systems with switching topologies and subject to time delays.

\section{Acknowledgements}

The authors wish to thank the anonymous reviewers for the careful reading of the manuscript and their insightful comments and suggestions. L. Ricciardi Celsi wishes to thank the Université Franco-Italienne/Università ItaloFrancese (UFI/UIF) for supporting his mobility from Italy to France during his PhD program.

\section{References}

D. Aeyels and F. De Smet. Emergence and evolution of multiple clusters of attracting agents. Physica D: Nonlinear Phenomena, 239(12):1026-1037, 2010.

R. Agaev and P. Chebotarev. On the spectra of nonsymmetric laplacian matrices. Linear Algebra and its Applications, 399:157-168, 2005.

C.O. Aguilar and B. Gharesifard. On almost equitable partitions and network controllability. In Proceedings of the 2016 ACC, pages 179-184. 2016.

S. Barbarossa, S. Sardellitti, and A. Farina. On sparse controllability of graph signals. In Proceedings of the IEEE CASSP, pages 4104-4108. 2016.

V.D. Blondel, J.M. Hendrickx, and J.N. Tsitsiklis. Continuous-time average-preserving opinion dynamics with opinion-dependent communications. SIAM Journal on Control and Optimization, 48(8):52145240, 2010.

D.M. Cardoso, C. Delorme, and P. Rama. Laplacian eigenvectors and eigenvalues and almost equitable partitions. European Journal of Combinatorics, 28: 665-673, 2007.

J.S. Caughman and J.J.P. Veerman. Kernels of directed graph laplacians. Electronic Journal of Combinatorics, 13(1):1-8, 2006.

Y. Chen, J. Lu, F. Han, and X. Yu. On the cluster consensus of discrete-time multi-agent systems. Systems and Control Letters, 60(7):517-523, 2011.

Y. Chen, W. Xiong, and F. Li. Convergence of infinite products of stochastic matrices: a graphical decomposition approach. IEEE Transactions on Automatic Control, 61(11):3599-3605, 2016.

C. Godsil and G.F. Royle. Algebraic graph theory. Springer Science \& Business Media, 2013.

A. Jadbabaie, J. Lin, and A.S. Morse. Coordination of groups of mobile autonomous agents using nearest neighbor rules. IEEE Transactions on Automatic Control, 48(6):988-1001, 2003.

J. Jin and Y. Zheng. Consensus of multi-agent system under directed network: A matrix analysis approach. In Proceedings of the 2009 IEEE ICCA 2009, pages 280-284. 2009.
J. Li, Z.H. Guan, and G. Chen. Multi-consensus of nonlinearly networked multi-agent systems. Asian Journal of Control, 17(1):157-164, 2015.

F. Lo Iudice, F. Garofalo, and F. Sorrentino. Structural permeability of complex networks to control signals. Nature Communications, 6, 2015.

S. Martini, M. Egerstedt, and A. Bicchi. Controllability analysis of multi-agent systems using relaxed equitable partitions. International Journal of Systems, Control and Communications, 2(1-3):100-121, 2010.

M. Mesbahi and M. Egerstedt. Graph theoretic methods in multiagent networks. Princeton University Press, 2010.

N. Monshizadeh, S. Zhang, and M. Kanat Camlibel. Disturbance decoupling problem for multi-agent systems: A graph topological approach. Systems and Control Letters, 76:35-41, 2015.

L. Moreau. Stability of multiagent systems with timedependent communication links. IEEE Transactions on Automatic Control, 50(2):169-182, 2005.

N. O'Clery, Y. Yuan, G.B. Stan, and M. Barahona. Observability and coarse graining of consensus through the external equitable partition. Physical Review E, 88, 2013.

A. Pietrabissa and L. Ricciardi Celsi. Discrete-time selfish routing converging to the Wardrop equilibrium. IEEE Transactions on Automatic Control, in press, 2018. doi: 10.1109/TAC.2018.2847602.

W. Ren and Y. Cao. Distributed Coordination of Multiagent Networks. Communications and Control Engineering Series, Springer-Verlag, London, 2011.

M.T. Schaub, N. O’Clery, Y.N. Billeh, J.C. Delvenne, R. Lambiotte, and M. Barahona. Graph partitions and cluster synchronization in networks of oscillators. Chaos, 26, 2016.

T. Vicsek, A. Czirk, E. Ben-Jacob, I. Cohen, and O. Shochet. Novel type of phase transition in a system of self-driven particles. Physical Review Letters, 75(6):1226-1229, 1995.

J.W. Yi, Y.W. Wang, and J.W. Xiao. Reaching cluster consensus in multi-agent systems. In Proceedings of the ICICIP 2011, pages 569-573. 2011.

J. Yu and L. Wang. Group consensus in multi-agent systems with switching topologies and communication delays. Systems and Control Letters, 59(6):340-348, 2010 .

S. Zhang, M. Cao, and M. Kanat Camlibel. Upper and lower bounds for controllable subspaces of networks of diffusively coupled agents. IEEE Transactions on Automatic Control, 59(3):745-750, 2014. 